\section{ORIGINAL RESEARCH}

X. Lv

C. Jiang

J. Zhang

Y. Li

Z. $\mathrm{Wu}$

\title{
Complications Related to Percutaneous Transarterial Embolization of Intracranial Dural Arteriovenous Fistulas in $\mathbf{4 0}$ Patients
}

\begin{abstract}
BACKGROUND AND PURPOSE: An increasing number of intracranial dural arteriovenous fistulas (DAVFs) are amenable to endovascular treatment with Onyx. However, reports on complications caused by this technique have been limited. We present the initial Beijing Tiantan Hospital experience with adverse events related to transarterial Onyx embolization for DAVFs.
\end{abstract}

MATERIALS AND METHODS: Between September 2005 and February 2008, a total of 40 patients with DAVFs were treated at our institute with Onyx-18. There were 11 women and 29 men with a mean age of 43.15 years (age range, 23-60 years). We reviewed the clinical presentation, angiographic features, treatment, and outcome.

\begin{abstract}
RESULTS: In 40 patients, total obliteration was achieved in 25 DAVFs (62.5\%), with the remaining 15 patients not cured with residual shunts. Complications occurred in 9 patients, 5 DAVFs were located at tentorium, 2 were located at the transverse-sigmoid sinus, 1 was found at the inferior petrosal sinus, and 1 was found at the cavernous sinus, leading to permanent disability in 3 patients (morbidity, $7.5 \%$ ). Complications included reflexive bradyarrhythmia in $3(7.5 \%)$ patients, hemifacial hypoesthesia in 3 (7.5\%) patients, hemifacial palsy in $2(5 \%)$ patients, posterior fossa infarction in $2(5 \%)$ patients, jaw pain in $1(2.5 \%)$ patient, microcatheter gluing in $1(2.5 \%)$ patient, hallucinations in $1(2.5 \%)$ patient, and Onyx migration in 1 (2.5\%) patient.
\end{abstract}

CONCLUSION: Although complete cure can be achieved by transarterial embolization with Onyx, the potential for serious complications exists with this procedure.

I

ntracranial dural arteriovenous fistulas (DAVFs) are defined as abnormal connections between an arterial feeder and a dural venous sinus or leptomeningeal vein, with the point of fistulization located within the dural leaflets. They compose $10 \%$ to $15 \%$ of all intracranial vascular malformations. ${ }^{1-3}$ Endovascular techniques allow both arterial and venous access to the site of the fistulous connection. ${ }^{4-18}$ DAVFs with leptomeningeal venous drainage or an associated venous varix are defined as high risk and are associated with a dismal natural history. ${ }^{2,19-24}$ High-risk fistulas typically present with hemorrhage or progressive neurologic deficits. ${ }^{13,25-36}$ Most DAVFs can be successfully managed with endovascular techniques. Transarterial Onyx (ev3, Irvine, Calif) embolization is now an established method of treatment of DAVFs. ${ }^{4,13,14,17,37,38}$ Onyx can often penetrate to the point of fistulization with distal ligation of multiple feeders. However, potential complications are associated with this procedure, and there is a lack of detailed information on such complications in the literature. In our study, we detailed our complications of transarterial embolization for DAVFs.

\section{Materials and Methods}

From February 2005 to February 2008, a total 40 patients with intracranial DAVFs were treated at Beijing Tiantan Hospital. The clinical records and angiographic studies of these patients were reviewed ret-

Received September 1, 2008; accepted after revision October 21

From the Beijing Neurosurgical Institute and Beijing Tiantan Hospital, Capital Medical University, Beijing, People's Republic of China.

Please address correspondence to Chuhan Jiang, MD, Beijing Neurosurgical Institute and Beijing Tiantan Hospital, Capital Medical University, Beijing, People's Republic of China, 6, Tiantan Xili, Beijing, 100050 Hebei, China; e-mail: ttyyzjb@sina.com

E indicates article with supplemental on-line table.

DOI 10.3174/ajnr.A1419 rospectively. They consisted of 11 women and 29 men, ranging in age from 23 to 60 years (mean, 43.15 years). Clinical follow-up data were supplemented by telephone interviews and graded according to the modified Rankin Scale (mRS). We performed the follow-up and Rankin grading. The mean duration of neurologic follow-up was 8 months (range, 1-16 months). Follow-up angiography was obtained in 29 patients at 5.4 months (range, 3-8 months) after the last procedure.

\section{Transarterial Technique}

All embolizations were performed with the patient under general anesthesia. Catheterization was performed with a transfemoral approach and use of standard coaxial techniques. The guiding catheter was flushed via a pressure bag with saline containing $2500 \mathrm{U}$ of heparin/L. If necessary, a second $5 \mathrm{~F}$ catheter was placed in the ipsilateral internal carotid artery or ipsilateral vertebral artery. DAVF feeders were superselectively catheterized with a dimethyl-sulfoxide-compatible microcatheter (Marathon; ev3). Once the microcatheter tip was in the desired position (as near as possible to the fistula), the injection of Onyx was carried out. Onyx is manufactured as Onyx-18, Onyx-20, and Onyx-34. In general, Onyx-18 is used for embolization in our series.

Single injections of Onyx were carried out for up to 90 minutes. Long injection times were possible because of the less adhesive nature of Onyx. During injections, we were able to pause, obtain an angiogram to assess fistula occlusion and the status of draining veins, and then continue with the injection. When any reflux of Onyx into the feeding pedicle or venous migration was noted, the injection was stopped for 1 to 2 minutes to allow for solidification, and then injection was continued. The Onyx then filled a different portion of the shunts, with no further reflux or filling of the vein. When Onyx did not fill in an antegrade fashion, the catheter was withdrawn. In some cases, where complete shunt occlusion was likely, we attempted to 


\section{Table 1: Symptoms of patients with DAVFs}

\begin{tabular}{lc}
\hline Symptoms & $n(\%)$ \\
\hline Intracranial hemorrhage & $16(40 \%)$ \\
Tinnitus/bruits & $10(25 \%)$ \\
Ocular symptoms & $11(27.5 \%)$ \\
Headaches & $4(10 \%)$ \\
Trigeminal neuralgia & $1(2.5 \%)$ \\
Hemihypoesthesia & $1(2.5 \%)$ \\
\hline
\end{tabular}

Note:-DAVF indicates dural arteriovenous fistula.

"push" the material across the arteriovenous shunts and into the proximal draining vein. For other DAVFs, the goal of embolization was to reduce DAVF shunts. Provocative testing was not performed.

\section{Results}

A total of 16 patients (40\%) presented with intracranial hemorrhage; 4 (10\%) had headaches without imaging evidence of bleeding; 11 (27.5\%) had ocular symptoms, including chemosis, ptosis, ophthalmoplegia, and hemianopsia; 10 (25\%) had tinnitus and intracranial bruits; 1 (2.5\%) had hemihypoesthesia; and $1(2.5 \%)$ had trigeminal neuralgia (Table 1$)$. Venous drainage patterns were classified according to the system described by Cognard et al. ${ }^{39}$ One patient had 1 type $V$ fistula, 18 patients had type IV, 3 had type III, 13 had type IIa fistulas, and 3 had type I fistulas.

All patients were treated with transarterial approach, with 1 patient treated with the additional transvenous approach. Three type I fistulas were treated because of symptoms of tinnitus and difficulty in transvenous treatment. Posttreatment angiography was performed routinely, and immediate angiographic obliteration was demonstrated in 25 patients $(62.5 \%)$. No additional treatments (transvenous approaches, surgery, and stereoradiologic surgery) were given to the 15 patients with residual shunt. Four patients with Cognard IV and 1 patient with Cognard V postembolization did not want to undergo another intervention because of they did not have symptoms; for other patients with Cognard I or II lesions, additional treatment was not felt to be necessary. Three residual fistulas were downgraded by treatment. Complications related to transarterial treatment occurred in 9 patients $(22.5 \%)$ and are summarized in on-line Table 1.

\section{Complications of Embolization}

Complications of embolization occurred in 5 patients $(12.5 \%)$. In 1 patient, a microcatheter was unable to be retrieved in the feeding vessel or nidus and was cut off in the groin without clinical sequelae. Pulmonary and cardiac Onyx migration occurred in 1 patient with left transverse-sigmoid sinus DAVF. Reflexive bradyarrhythmia associated with Onyx injection developed in 2 patients with tentorial DAVF and in 1 patient with a DAVF involving the inferior petrosal sinus. Of 40 DAVFs, embolization of the feeders of the trigeminal and facial nerves associated with Onyx reflux occurred in 2 patients with hemifacial hypoesthesia and hemifacial palsy and in 1 patient with hemifacial hypoesthesia. One patient exhibited vertigo, diplopia, blepharoptosis, ataxia, hemihypoesthesia, gustatory loss, and deterioration of ataxia after a second procedure (Patient 1). On postprocedure day 3, MR imaging examination was obtained and demonstrated infarctions of the left cerebellar hemisphere, the left side of the mesence- phalic tegmentum, and the left middle cerebellar peduncle. One patient had blepharoptosis, diplopia, and mild ataxia of the left leg after embolization. CT on postprocedure day 3 revealed a small infarction in the left cerebellar hemisphere. Jaw pain occurred in 1 patient, caused by, we believe, vasospasm of the internal maxillary artery. Also, 1 patient complained of hallucinations the next day after the procedure.

\section{Clinical Outcome}

Follow-up angiography was performed in 29 patients and confirmed angiographic cure in 21 patients. All patients returned to independent clinical status during the follow-up period (range, 1-16 months; mean, 8 months). Clinical outcome was graded according to the mRS, modified as follows: 0 , neurologically intact ( $n=37$ [92.5\%]); and 1, mild hemiparesis, cranial nerve palsy, or other deficit that does not interfere with daily functioning or work $(n=3[7.5 \%])$. The overall rate of permanent morbidity was $7.5 \%$.

\section{Illustrative Cases}

Case 1. A 28-year-old man with a left sigmoid sinus DAVF (Cognard type I) was embolized with Onyx-18 (Fig. 1). Transarterial embolization was performed through the left occipital artery. Onyx was carefully injected with fluoroscopic guidance. Care was taken to avoid any arterial Onyx reflux or venous Onyx migration. The intracranial circulation was intact, the same as was seen on the preprocedure angiogram. The postembolization angiogram showed a remaining small part of the arteriovenous shunt. The patient was discharged on day 3 after the procedure. From a neurologic standpoint, the patient was intact at the end of the procedure. However, approximately 9 months after the procedure, he experienced cardiac palpitations. A chest radiographic examination was performed, which showed multiple small fragments of Onyx located in the pulmonary artery and right cardiac ventricle (Fig. $1 D$ ). Cardiac surgery followed with complete removal of the fragment of Onyx that was located intracardiacally. The patient had an uneventful recovery.

Case 8. This 41-year-old man presented with a Cognard type IV DAVF involving the left cerebellar tentorium (Fig. 2). The left posterior cerebral artery was wedged, and the DAVF was embolized completely with Onyx-18. During Onyx injection, reflux via the anastomosis between the collicular arteries of the posterior cerebral artery (PCA) and superior cerebellar artery (SCA) was found. Angiography after embolization showed complete obliteration of the lesion, and the patient exhibited left blepharoptosis and mild ataxia of the left leg on the first day after the procedure. Postprocedure CT scanning revealed left cerebellar infarction. At 3-month follow-up, he could walk by himself but still had blepharoptosis.

Case 4. This 60-year-old woman presented with blurred vision of both eyes and pulsatile tinnitus on the right side (Fig. 3). Ophthalmic examination revealed normal visual acuity and normal intraocular pressure and motility. Ophthalmoscopic examination revealed no papilledema. Visual field testing demonstrated bilateral inferior hemianopsia. MR angiography showed the right transverse sinus at the arterial phase. Subsequent digital subtraction angiography confirmed DAVF of the right sigmoid sinus, occlusion of the right sigmoid sinus, and reversal of the flow. The patient was treated with transar- 

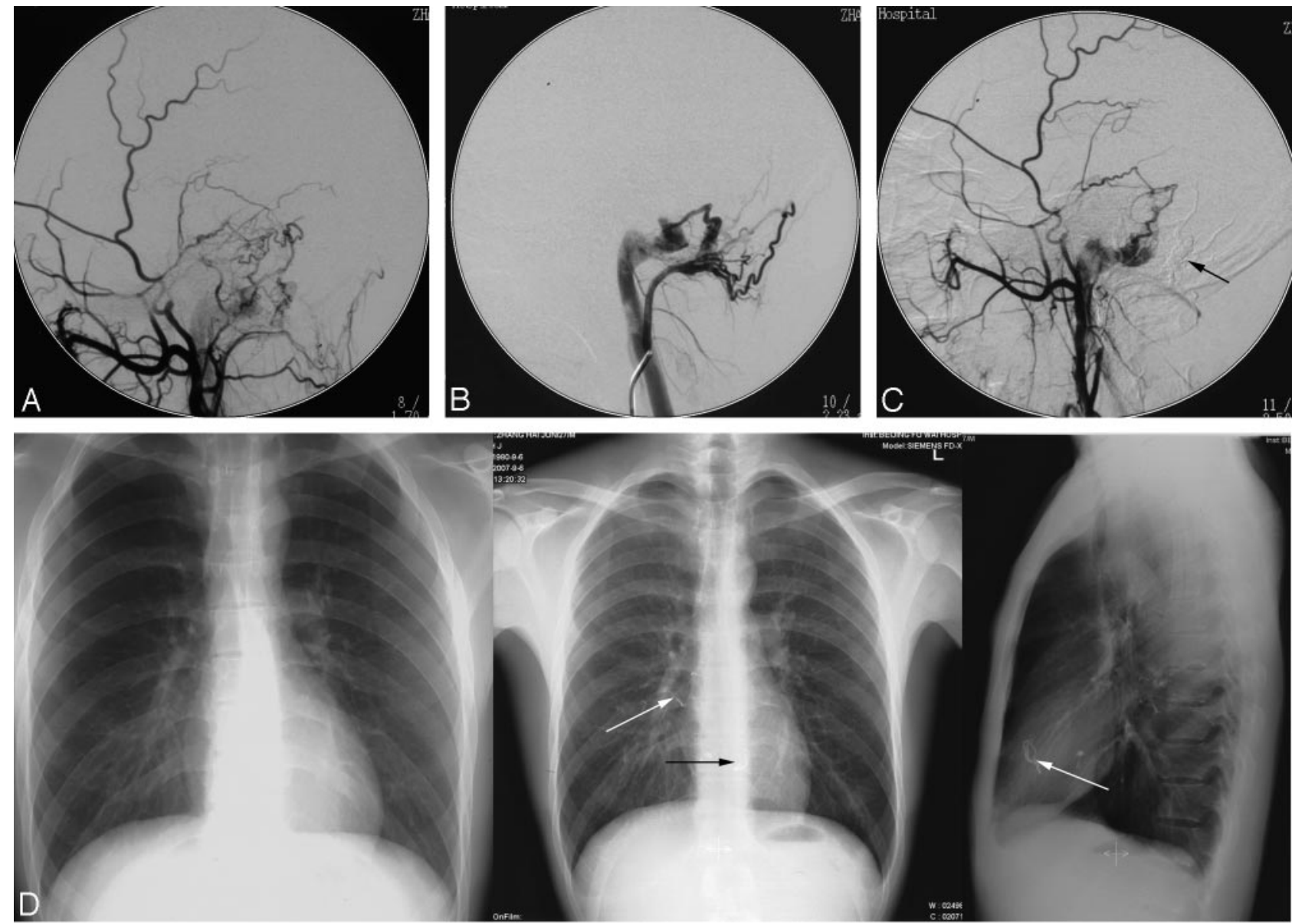

Fig 1. A 28-year-old man presented with intracranial bruits caused by a left sigmoid sinus DAVF. The fistula was supplied by feeders from the ipsilateral external carotid artery. The left external carotid angiograms $(A$ and $B$ ) show a left sigmoid sinus DAVF (Cognard type I). $C$, the DAVF shows incomplete obliteration with a single Onyx injection through the left occipital artery. Note Onyx cast (arrows). D, Results of a thoracic radiograph (left) were normal while the patient was being admitted. Thoracic radiographs 9 months later. A frontal view (middle) and lateral view (right) show the Onyx migration in the heart and pulmonary artery (arrow).

terial embolization with Onyx-18 (Fig. 4). On the first day after the procedure, she complained of visual hallucinations. She described seeing "beautiful pictures on the walls and ceiling of my hospital room." She recognized these symptoms as unreal and denied the possibility of dreams. The possible causes of neurologic deficits such as electrolyte imbalance were ruled out by relevant tests, so a diagnosis of venous ischemia of the left occipital lobe was made. Her visual hallucinations intermittently appeared for the next 2 days and then disappeared. She returned to her previous employment with no neurologic deficits.

\section{Discussion}

Our initial experience with use of Onyx for embolization of intracranial DAVFs is encouraging, with complete obliteration with transarterial embolization in $62.5 \%$. In most patients, 1 embolization session was sufficient to obtain a complete obliteration or sufficient arteriovenous shunt reduction. We believe that Onyx has several advantages compared with n-butyl-2 cyanoacrylate. The introduction of Onyx in most DAVF embolizations in our practice has transformed the embolization of DAVFs from a rather unpredictable intervention into a controlled procedure. In the external carotid territory, a longer reflux of the Onyx around the tip of the microcatheter can be achieved more safely compared with the pial arteries, provided that the microcatheter is placed sufficiently distally as long as the operator is confident that he or she will be able to retrieve the microcatheter. This long reflux may create sufficient proximal flow arrests to enable better distal penetration. ${ }^{4,13,14,17,37,38}$ Consequently, when used in DAVFs, it may be more realistic to consider Onyx as a potential permanent embolic agent rather than expecting it to be surgically removed. In smaller arteriovenous fistulas, cure can be potentially attained with Onyx injection only.

Although percutaneous transarterial embolization offers an easy, safe access in most cases of DAVFs, it may occasionally lead to the complications discussed: microcatheter gluing, pulmonary and cardiac Onyx migration, reflexive bradyarrhythmia, damage of cranial nerves V and VII, cerebellar infarction, hallucinations, and jaw pain. The most serious complications are brain infarction and migration of the Onyx into other organs via the arteriovenous shunts, such as the lungs and heart. Difficulty in microcatheter retrieval, reflexive bradyarrhythmia during Onyx injection, and trigeminal and facial nerve deficits caused by Onyx reflux have been described in the previous literature. ${ }^{13,38}$

\section{Reflexive Bradyarrhythmia}

This reproducible response occurred in 2 patients with tentorial DAVFs, Cognard IV. When stimulation causes trigemino- 

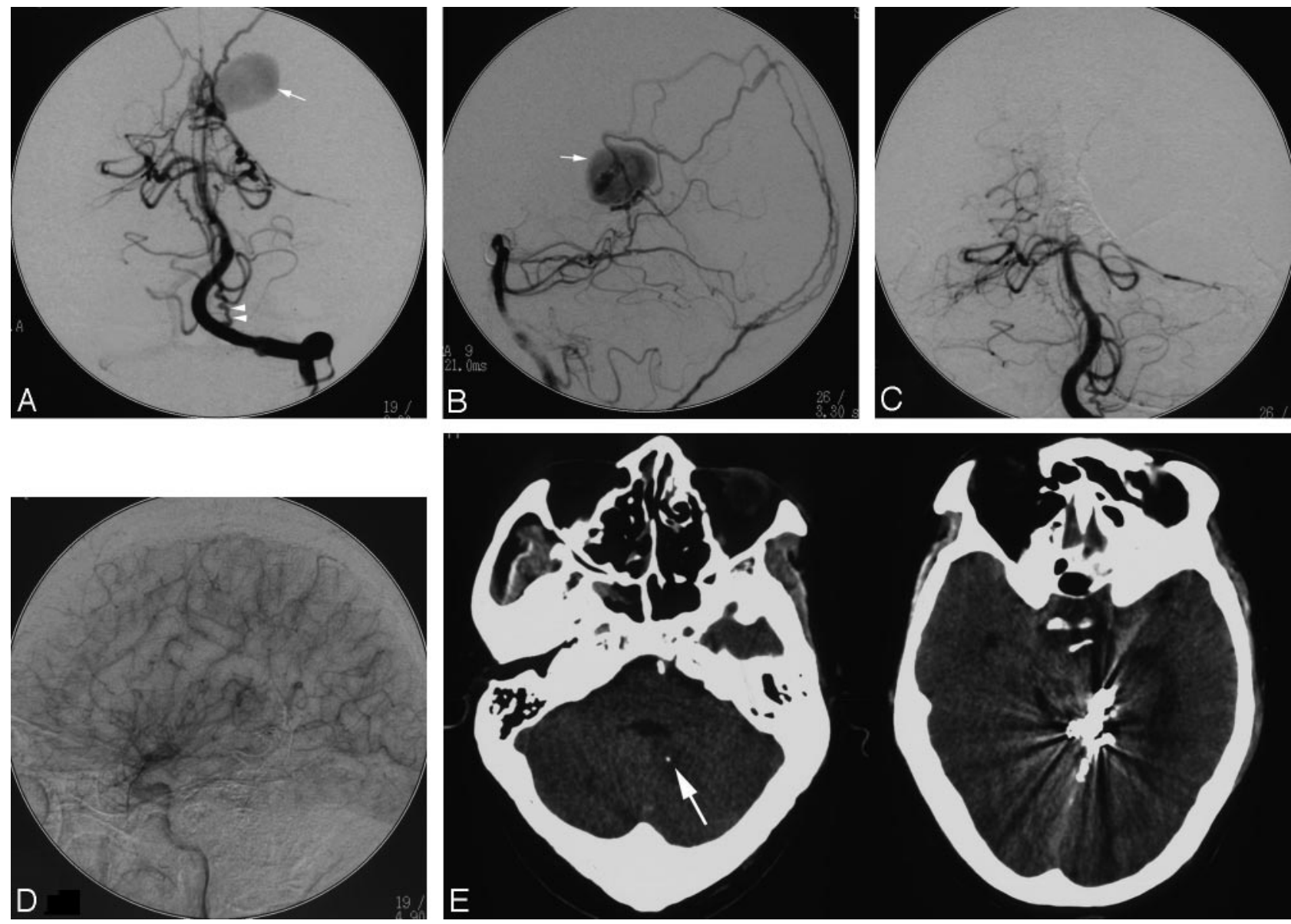

Fig 2. A 41-year-old man presented with a DAVF involving the left cerebellar tentorium. Left vertebral angiograms, frontal projection $(A)$ and lateral projection $(B)$, show a DAVF involving the left cerebellar tentorium supplied by the posterior meningeal artery and branches of the left posterior cerebral artery with a venous aneurysm (white arrow); the posterior meningeal artery was tortuous (double arrowheads). After embolization, a left vertebral angiogram, anteroposterior projection $(C)$ revealed complete obliteration of the DAVF and P2 segment of the left PCA. Left common carotid angiogram, lateral projection $(D)$, shows collateral flow to the left PCA territory. CT scanning $(E)$ on the first day after the procedure demonstrated infarction in the left cerebellum (white arrow). The hyperdense areas (prepontine and premesencephalic cistern) are Onyx cast.

cardiac reflex (TCR), treatment of hemodynamic instability consists of ceasing Onyx injection, and anticholinergic drugs should be administered. ${ }^{38}$ In 1 patient of our series, although Onyx was permeated well and cure could be achieved if Onyx injection was continued, TCR occurred and the heart rate decreased to $45 \mathrm{bpm}$. We had to cease Onyx injection, and atropine was administered transvenously. After the patient's heart rate returned to $70 \mathrm{bpm}$, we continued the injection of Onyx, but the Onyx could not be pushed in an antegrade fashion. Because of this complication, we suggest that anticholinergic drugs be given prophylactically because they can affect the outcome of embolization.

\section{Neuropathy of Cranial Nerves V and VII}

Immediately adjacent to the foramen spinosum, the middle meningeal artery gives off a short branch, which divides into the petrosal artery laterally to the geniculate ganglion and a cavernous branch to the trigeminal ganglion medially. We believe that occlusion of the cavernous branch and petrosal branch occurring as the Onyx refluxed into the pedicle of the middle meningeal artery may result in trigeminal and facial nerve deficits; therefore, Onyx should not be refluxed to the foramen spinosum level. This observation can be supported by the patients in our series who complained of hemifacial numbness and dull sensation to touch along the innervation of the second and third trigeminal divisions. In our series, $1 \mathrm{pa}-$ tient with cavernous DAVF experienced hemifacial hypoesthesia, and 2 patients who had tentorial DAVF and DAVF involving superior petrosal sinus experienced hemifacial hypoesthesia and hemifacial palsy after embolization. During follow-up, facial function and sensation of the patient with DAVF involving the superior petrosal sinus did not recover completely. We believe that the poor recovery of facial function and sensation was the result of the vascular insult into the facial nerve. In addition, the embolization procedure itself may have caused additional vascular insult to the nerve.

\section{Onyx Migration}

In this series, Onyx migrated into the heart and pulmonary artery in a patient with sigmoid sinus DAVF, which has not been detailed in the endovascular treatment of DAVFs. Migration of Onyx is presumed to happen only when the liquid embolic agent is in its liquid state during injection. ${ }^{40}$ Although we inject Onyx under biplane fluoroscopy, we did not observe migration of this agent during the procedure. We considered that the Onyx-18 may migrate invisibly under biplane fluoroscopy because of fast shunt. We recommend using a higher concentration of Onyx (Onyx-34) to promote faster solidifi- 

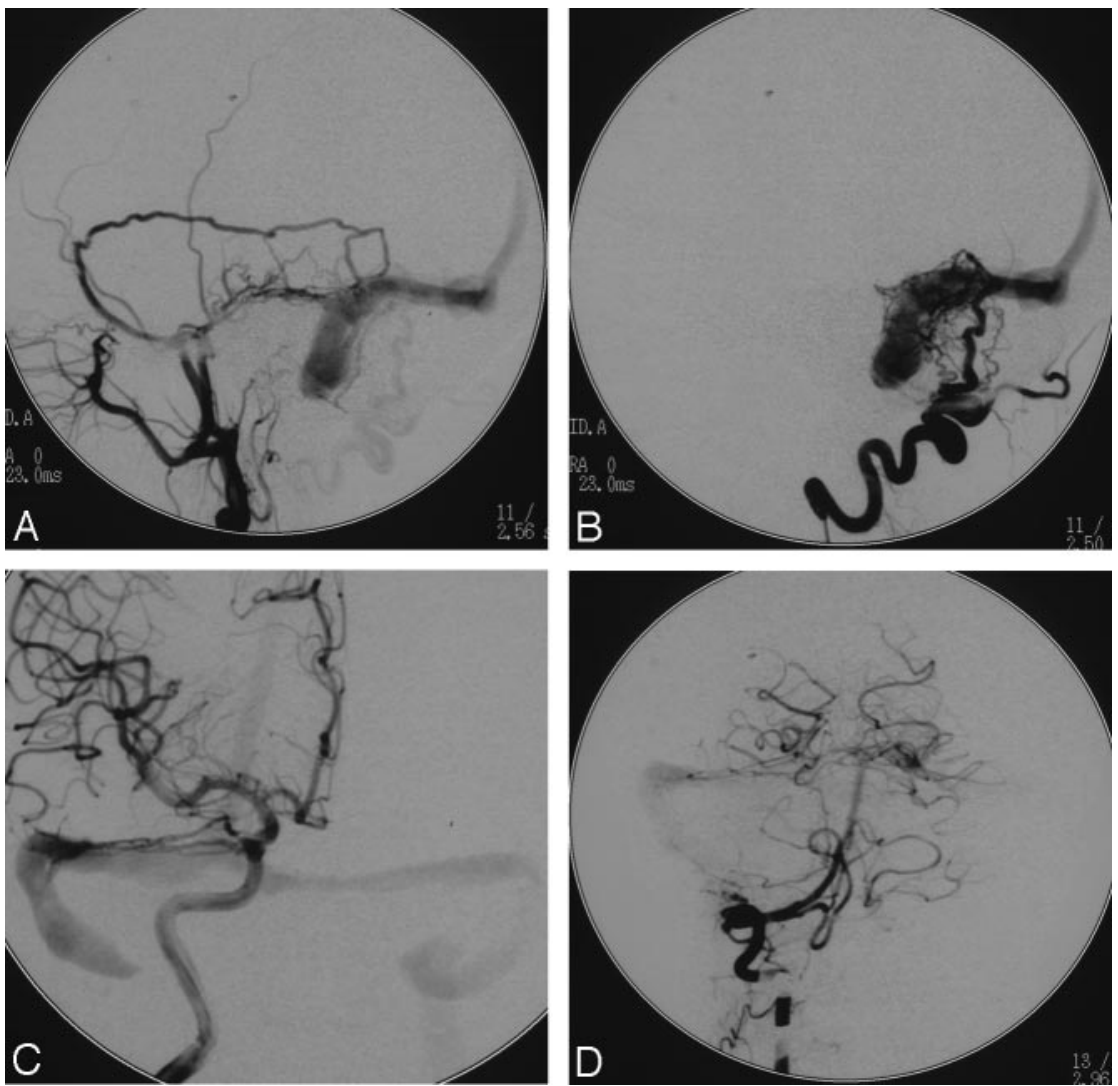

Fig 3. Right external carotid angiograms $(A$ and $B)$ show the DAVF supplied by the branches of the right middle meningeal artery and right occipital artery, with draining into the left sigmoid sinus. Note superior sagittal sinus reflux and occlusion of the right sigmoid sinus. Right internal carotid angiogram $(C)$ and right vertebral angiogram (D) show that the DAVF was also fed by the suppliers from the right internal carotid artery and the right posterior cerebral artery.

cation. The intact neurologic status of the patient after the procedure further ruled out migration of the Onyx into the cerebral circulation during the procedure. Nine months later, because of the presence of a demonstrable Onyx cast in the pulmonary artery and the right cardiac ventricle, it is highly likely it embolized during the procedure. It is unlikely that the Onyx migrated through the arteriovenous shunt during the postoperative period because the volume of Onyx cast seems larger than the arteriovenous shunt. The Onyx may also have migrated to the jugular vein early on and subsequently traveled to the heart and pulmonary artery.

\section{Hallucinations}

In the case of our patient who experienced hallucinations, the sigmoid sinus DAVF caused bilateral inferior hemianopsia. On the first day after treatment, the patient complained of vivid visual hallucinations, suggesting a diagnosis of hallucinations. The hallucinations disappeared spontaneously. These findings indicated that hallucinations might be a manifestation of venous ischemia after transarterial treatment. The blood shunted into an occluded sigmoid sinus and reflux blood flowed through the sagittal sinus. It can further be assumed that this arterialized flow led to an increased venous pressure and restricted the normal parenchymal venous drainage, probably causing venous ischemia of the left occipital lobe.

It is likely that the anopsia was caused by disturbed venous drainage of the occipital lobe. Thus, normal venous drainage of the left inferior calcarine cortex was probably compromised, accounting for the visual field loss. ${ }^{41}$ In DAVFs with antegrade flow obstruction and reflux into the superior sagittal sinus, straight sinus, or cortical veins, the retrograde transmission of pressure results in intracranial venous ischemia and the resultant congestion in return impairs parenchymal venous damage, thereby causing ischemia. ${ }^{29,42}$ This finding may have been caused by the coexistence of increased venous pressure with resultant decreased cerebral perfusion pressure or by a posttreatment reperfusion phenomenon.

\section{Cerebellar Infarction}

Two patients with tentorial DAVFs of Cognard IV in our series were diagnosed with cerebellar infarctions after embolization. Clear visualization of the SCA on the diseased side had the duplication in 1 patient. The inferior trunk was catheterized and occluded with Onyx. Although the inferior trunk of the SCA gives rise to perforators in $27 \%$ of patients, ${ }^{6}$ it cannot be used as an approach for Onyx injection. The second patient was treated with Onyx embolization via the tentorial artery of the left posterior cerebral artery. We suggest that Onyx may reflux via the anastomosis between the collicular arteries of the PCA and SCA and causes occlusion of the medial SCA branch.

\section{Conclusions}

Our study shows that transarterial treatment of DAVFs carries a substantial risk for significant and long-term injury. That is why such treatment should be restricted to high-grade fistulas, 

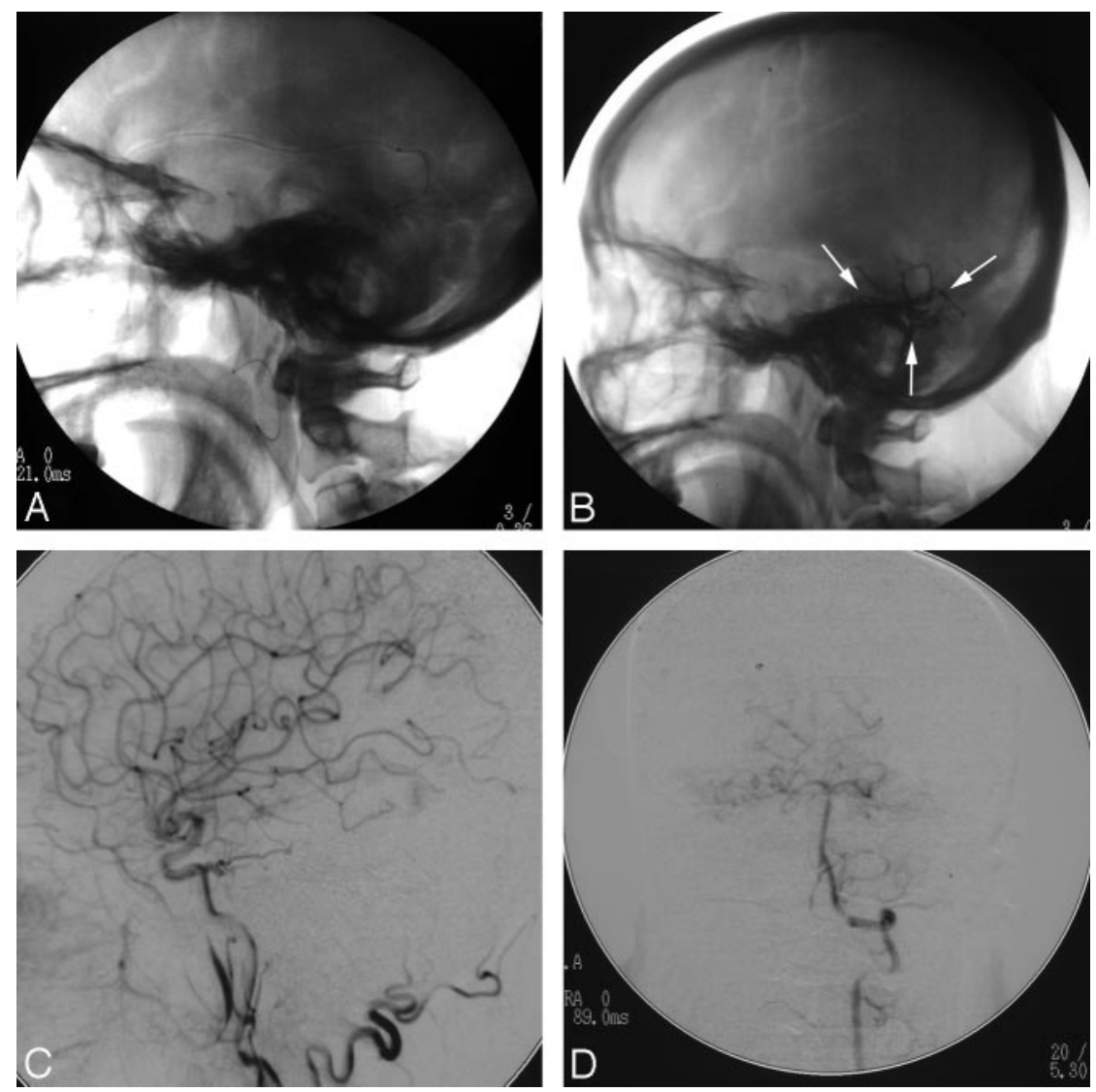

Fig 4. Fluoroscopic image $(A)$ showed that the right middle meningeal artery was catheterized. Fluoroscopic image $(B)$ after embolization showed the Onyx cast (arrows). Right common carotid angiogram $(C)$ and left vertebral angiogram $(D)$ revealed complete obliteration of the DAVF.

which carry a poor prognosis without treatment and in which alternative treatments are less safe. Complications such as microcatheter gluing, pulmonary and cardiac Onyx migration, reflexive bradyarrhythmia, damage of cranial nerves $\mathrm{V}$ and VII, midbrain and cerebellar infarction, hallucinations, and jaw pain can be caused by this procedure. Physicians performing the procedure and patients should understand these issues.

\section{References}

1. Kim MS, Han DH, Kwon OK, et al. Clinical characteristics of dural arteriovenous fistula. J Clin Neurosci 2002;9:147-55

2. King WA, Martin NA. Intracerebral hemorrhage due to dural arteriovenous malformations and fistulae. Neurosurg Clin N Am 1992;3:577-90

3. Sarma D, ter Brugge K. Management of intracranial dural arteriovenous shunts in adults. Eur J Radiol 2003;46:206-20

4. Arat A, Inci S. Treatment of a superior sagittal sinus dural arteriovenous fistula with Onyx: technical case report. Neurosurgery 2006;59(1 Suppl 1): ONSE169-70; discussion ONSE169-70

5. Barnwell SL, Halbach VV, Higashida RT, et al. Complex dural arteriovenous fistulas. Results of combined endovascular and neurosurgical treatment in 16 patients. J Neurosurg 1989;71:352-58

6. Biondi A, Milea D, Cognard C, et al. Cavernous sinus dural fistulae treated by transvenous approach through the facial vein: report of seven cases and review of the literature. AJNR Am J Neuroradiol 2003;24:1240-46

7. Caragine LP, Halbach VV, Dowd CF, et al. Parallel venous channel as the recipient pouch in transverse/sigmoid sinus dural fistulae. Neurosurgery 2003;53:1261-67

8. Dawson RC 3rd, Joseph GJ, Owens DS, et al. Transvenous embolization as the primary therapy for arteriovenous fistulas of the lateral and sigmoid sinuses. AJNR Am J Neuroradiol 1998;19:571-76

9. Fok KF, Agid R, Souza MP, et al. Thrombosis of aggressive dural arteriovenous fistula after incomplete embolization. Neuroradiology 2004;46:1016-21

10. Kincaid PK, Duckwiler GR, Gobin YP, et al. Dural arteriovenous fistula in children: endovascular treatment and outcomes in seven cases. AJNR Am J Neuroradiol 2001;22:1217-25
11. Klisch J, Huppertz HJ, Spetzger U, et al. Transvenous treatment of carotid cavernous and dural arteriovenous fistulae: results for 31 patients and review of the literature. Neurosurgery 2003;53:836-57

12. Liu A, Lv X, Li Y, et al. Traumatic middle meningeal artery and fistula formation with the cavernous sinus: case report. Surg Neurol 2008;70:660-63

13. Lv X, Jiang C, Li Y, et al. Transarterial embolization of tentorial dural arteriovenous fistulas with Onyx 18. Neuroradiol J 2008;21:406-14

14. Lv X, Li Y, Wu Z. Endovascular treatment of anterior cranial fossa dural arteriovenous fistula. Neuroradiology 2008;50:433-37

15. Ng PP, Higashida RT, Cullen S, et al. Endovascular strategies for carotid cavernous and intracerebral dural arteriovenous fistulas. Neurosurg Focus 2003;15:ECP1

16. Ng PP, Halbach VV, Quinn R, et al. Endovascular treatment for dural arteriovenous fistulae of the superior petrosal sinus. Neurosurgery 2003;53:25-33

17. Rezende MTS, Poitin M, Mounayer C, et al. Dural arteriovenous fistula of the lesser sphenoid wing region treated with Onyx: technical note. Neuroradiology 2006;48:130-34

18. van Rooij WJ, Sluzewski M, Beute GN. Tentorial artery embolization in tentorial dural arteriovenous fistulas. Neuroradiology 2006;48:737-43

19. da Costa LB, Terbrugge K, Farb R, et al. Surgical disconnection of cortical venous reflux as a treatment for Borden type II dural arteriovenous fistulae. Acta Neurochir (Wien) 2007;149:1103-08

20. Flynn TH, McSweeney S, O'Connor G, et al. Dural AVM supplied by the ophthalmic artery. Br J Neurosurg 2007;21:414-16

21. Kakarla UK, Deshmukh VR, Zabramski JM, et al. Surgical treatment of highrisk intracranial dural arteriovenous fistulae: clinical outcomes and avoidance of complications. Neurosurgery 2007;61:447-59

22. Pelz DM, Lownie SP, Fox AJ, et al. Intracranial dural arteriovenous fistulae with pial venous drainage: combined endovascular-neurosurgical therapy. Can J Neurol Sci 1997;24:210-18

23. Vougioukas VI, Coulin CJ, Shah M, et al. Benefits and limitations of image guidance in the surgical treatment of intracranial dural arteriovenous fistulas. Acta Neurochir (Wien) 2006;148:145-53

24. Zink WE, Meyers PM, Connolly ES, et al. Combined surgical and endovascular management of a complex posttraumatic dural arteriovenous fistula of the tentorium and straight sinus. J Neuroimaging 2004;14:273-76

25. Datta NN, Rehman SU, Kwok JC, et al. Reversible dementia due to dural arteriovenous fistula: a simple surgical option. Neurosurg Rev 1998;21:174-76 
26. Hurst RW, Marcotte P, Raps EC, et al. Dural arteriovenous fistulas involving the superior sagittal sinus: acute presentation with intracranial hemorrhage. Surg Neurol 1998;49:42-46

27. Ito M, Sonokawa $\mathrm{T}$, Mishina $\mathrm{H}$, et al. Reversible dural arteriovenous malformation-induced venous ischemia as a cause of dementia: treatment by surgical occlusion of draining dural sinus: case report. Neurosurgery 1995;37: 1187-92

28. Iwanuro Y, Nakahara I, Higashi T, et al. Tentorial dural arteriovenous fistula presenting symptoms due to mass effect on the dilated draining vein: case report. Surg Neurol 2006;65:511-15

29. Kim MS, Han DH, Han MH, et al. Posterior fossa hemorrhage caused by dural arteriovenous fistula: case reports. Surg Neurol 2003;59:512-17

30. Lawton MT, Chun J, Wilson CB, et al. Ethmoidal dural arteriovenous fistulae: an assessment of surgical and endovascular management. Neurosurgery 1999;45:805-11

31. Magot A, Desal H, Wiertlewski S, et al. Dural arteriovenous fistula. A rare cause of treatable dementia. Rev Neurol (Paris) 2004;160(4 Pt 1):425-33

32. Nakai K, Umezawa H, Kohyama S, et al. Dural arteriovenous fistula presenting as acute-onset dementia: a case report. No Shinkei Geka 1997;25:349-54

33. Oishi $\mathrm{H}$, Horinaka N, Shmizu $\mathrm{T}$, et al. Case of intracranial dural arteriovenous fistula presenting with brainstem infarction. No Shinkei Geka 2005;33: 1095-99

34. Rose $\mathrm{D}$, Devin $\mathrm{KB}$, Van $\mathrm{H}$, et al. Trigeminal neuralgia in a patient with a dural arteriovenous fistula in Meckel's cave: case report. Neurosurgery 2003;53. 216-21

35. Tomak PR, Cloft HJ, Kaga A, et al. Evolution of the management of tentorial dural arteriovenous malformations. Neurosurgery 2003;52:750-62

36. van Dijk JM, TerBrugge KG, Willinsky RA, et al. Selective disconnection of cortical venous reflux as treatment for cranial dural arteriovenous fistulas. J Neurosurg 2004;101:31-35

37. Lv X, Li Y, Liu A, et al. Transarterial embolization of dural arteriovenous fistulas of the anterior cranial fossa Onyx-18: case report. Neuroradiol 2007;20:53-59

38. Lv X, Li Y, Lv M, et al. Trigeminocardiac reflex in embolization of intracranial dural arteriovenous fistula. AJNR Am J Neuroradiol 2007;28:1769-70

39. Cognard C, Gobin YP, Pierot L, et al. Cerebral dural arteriovenous fistulas: clinical and angiographic correlation with a revised classification of venous drainage. Radiology 1995;194:671-80

40. Weber W, Siekmann R, Kis B, et al. Treatment and follow-up of 22 unruptured wide-necked intracranial aneurysms of the internal carotid artery with Onyx HD 500. AJNR Am J Neuroradiol 2005;26:1909-15

41. Benndorf G, Schmidt S, Sollmann WP, et al. Tentorial dural arteriovenous fistula presenting with various visual symptoms related to anterior and posterior visual pathway dysfunction: case report. Neurosurgery 2003;53:222-27

42. Ito $\mathrm{M}$, Sonokawa $\mathrm{T}$, Mishina $\mathrm{H}$, et al. Dural arteriovenous malformation manifesting as tic douloureux. Surg Neurol 1996;45:370-77 